\title{
Erratum to: Iron Deficiency Without Anemia Is Associated with Anger and Fatigue in Young Japanese Women
}

\author{
Takako Sawada $^{1} \cdot$ Aki Konomi $^{2} \cdot$ Katsuhiko Yokoi $^{3}$
}

Published online: 21 October 2015

(C) Springer Science+Business Media New York 2015

Erratum to: Biol Trace Elem Res (2014) 159:22-31

DOI 10.1007/s12011-014-9963-1

The authors wish to indicate the following corrections to the referenced article. Under Table 1, the IDNA value 11.6 \pm 2.5 (28) and $P$ value 0.307 for Menarche (years) are incorrect and should be corrected to $12.0 \pm 1.1$ (27) and 0.608 , respectively. Also, under Table 4, the quartiles value $19(10.7,39.5)$ for Overall, A-R is incorrect and should be corrected to $19(10.8,39.5)$. The corrected tables are shown below.

The online version of the original article can be found at http://dx.doi.org/ 10.1007/s12011-014-9963-1.

Katsuhiko Yokoi

yokoi@seitoku.ac.jp

1 Faculty of Food and Nutritional Sciences, Toyo University,

Gunma, Japan

2 Faculty of Health and Medical Sciences, Teikyo Heisei University, Tokyo, Japan

3 Department of Human Nutrition, Seitoku University Graduate School, Matsudo, Chiba 271-8555, Japan 
Table 1 Characteristics of the normal, IDNA, and IDA subjects

\begin{tabular}{|c|c|c|c|c|}
\hline & Normal & IDNA & IDA & $\begin{array}{l}\text { ANOVA } \\
P \text { value }\end{array}$ \\
\hline Age (years) & $19.4 \pm 0.6^{\mathrm{a}}(36)$ & $19.9 \pm 1.0^{\mathrm{b}}(29)$ & $19.8 \pm 1.1^{\mathrm{a}, \mathrm{b}}(10)$ & 0.028 \\
\hline Body height (m) & $1.58 \pm 0.06(36)$ & $1.59 \pm 0.06(29)$ & $1.57 \pm 0.07(10)$ & 0.554 \\
\hline Body weight $(\mathrm{kg})$ & $54.2 \pm 8.5(36)$ & $52.0 \pm 6.6(29)$ & $55.3 \pm 8.4(10)$ & 0.409 \\
\hline Body mass index ${ }^{1}\left(\mathrm{~kg} / \mathrm{m}^{2}\right)$ & $21.8 \pm 3.3(36)$ & $20.7 \pm 2.4(29)$ & $22.6 \pm 3.6(10)$ & 0.141 \\
\hline Systolic blood pressure (mmHg) & $101.3 \pm 9.0(35)$ & $105.1 \pm 9.4(28)$ & $106.0 \pm 14.7(10)$ & 0.227 \\
\hline Diastolic blood pressure (mmHg) & $65.6 \pm 8.9(35)$ & $65.8 \pm 7.2(28)$ & $67.2 \pm 7.7(10)$ & 0.855 \\
\hline Pulse (counts/min) & $74.3 \pm 9.5(35)$ & $76.6 \pm 9.1(28)$ & $79.5 \pm 17.2(10)$ & 0.356 \\
\hline Sublingual temperature $\left({ }^{\circ} \mathrm{C}\right)$ & $36.3 \pm 0.4(34)$ & $36.3 \pm 0.5$ (28) & $36.6 \pm 0.6(10)$ & 0.173 \\
\hline Birth weight (g) & $2938 \pm 561^{\mathrm{a}}(32)$ & $3289 \pm 631^{\mathrm{b}}(21)$ & $3399 \pm 314^{\mathrm{b}}(8)$ & 0.034 \\
\hline Menarche (years) & $12.3 \pm 1.3(34)$ & $12.0 \pm 1.1(27)$ & $12.1 \pm 0.9(9)$ & 0.608 \\
\hline Menstrual duration (days) & $5.5 \pm 1.0(31)$ & $5.9 \pm 1.2(29)$ & $6.0 \pm 1.3(10)$ & 0.284 \\
\hline Menstrual cycle (days) & $29.0 \pm 4.3(26)$ & $29.1 \pm 3.0(27)$ & $28.6 \pm 4.9(9)$ & 0.942 \\
\hline
\end{tabular}

Results are presented as means $\pm \mathrm{SD}(n)$. An overall difference between the groups was determined by one-way ANOVA. If the one-way ANOVA was significant $(P<0.05)$, differences between individual groups were estimated by using Fisher's protected least significant difference test $I D N A$ iron deficiency without anemia, IDA iron deficiency anemia

${ }^{1}$ Body mass index (BMI) was calculated as weight $(\mathrm{kg})$ divided by the square of height $(\mathrm{m})$

Table 4 Comparison of the somatic, mental and overall scores in the CMI-J for the normal, IDNA, and IDA subjects

\begin{tabular}{|c|c|c|c|c|c|c|c|}
\hline & \multicolumn{2}{|c|}{ Normal $(n=36)$} & \multicolumn{2}{|c|}{ IDNA $(n=29)$} & \multicolumn{2}{|l|}{ IDA $(n=10)$} & \multirow{2}{*}{$\begin{array}{l}\text { Kruskal-Wallis test } \\
P \text { value }\end{array}$} \\
\hline & Mean \pm SD & Median (quartiles) & Mean \pm SD & Median (quartiles) & Mean \pm SD & Median (quartiles) & \\
\hline Somatic, A-L & $19.1 \pm 15.1$ & $15.5(7.8,25.3)$ & $22.7 \pm 11.3$ & $19(16,31)$ & $18.0 \pm 10.7$ & $13.5(9,27.5)$ & 0.184 \\
\hline Mental, M-R & $8.1 \pm 8.3^{\mathrm{a}}$ & $5(2,12.5)$ & $12.5 \pm 9.3^{\mathrm{b}}$ & $8(5,19)$ & $7.7 \pm 10.9^{\mathrm{ab}}$ & $4.5(3,6)$ & 0.024 \\
\hline Overall, A-R & $27.3 \pm 22.7$ & $19(10.8,39.5)$ & $35.2 \pm 18.6$ & $33(21,42)$ & $25.7 \pm 18.4$ & $16.5(13.3,37)$ & 0.070 \\
\hline
\end{tabular}

IDNA iron deficiency without anemia, IDA iron deficiency anemia

a,b Values not sharing common superscript letters are significantly different $(P<0.05)$ by the Dwass-Steel-Chritchlow-Fligner test 